\title{
A Case of Bladder Cancer Found during a Workup for Urge Incontinence
}

\author{
Yun-Seob Song, Kyu-Hyung Cho, Keun-Woo Kim, Jong-Hyun Yoon, Seung-Hwan Doo, \\ Won-Jae Yang, Joo-Young Cho', Dong-Wha Lee ${ }^{2}$ \\ Departments of Urology, ${ }^{1}$ Gastroenterology, ${ }^{2}$ Pathology, Soonchunhyang University College of Medicine, Seoul, Korea
}

Irritative urinary symptoms may suggest the possibility of bladder cancer. We report a case of metastatic bladder cancer that was discovered during a workup for urge incontinence in a 65-year-old woman with a history of stomach cancer. She had a medical history of gastrectomy due to stomach cancer 4 years previously. The patient complained of urgency unresponsive to anticholinergic therapy. Cystoscopy revealed the presence of suspicious bladder mucosal lesions that were biopsied. The pathology was consistent with metastatic signet-ring cell adenocarcinoma. This case suggests that irritative urinary symptoms can be the first clinical manifestation in patients with bladder cancer.

Key Words: Urge incontinence; Bladder cancer; Stomach cancer

In Korea, bladder cancer is the sixth most frequent neoplasia in men and the second most prevalent urogenital neoplasia. Bladder cancer can be primary or metastatic.

Metastatic cancer is rare and constitutes approximately $1 \%$ of bladder cancer [1]. Signs and symptoms resulting from bladder involvement are present in approximately $20 \%$ of cases [2]. It is not easy to suggest metastatic bladder cancer in patients who complain of irritative urinary symptoms. We report a case of a 65-year-old woman with bladder metastasis of stomach cancer who underwent a previous gastrectomy due to stomach cancer 3 years before.

\section{Case}

A 65-year-old woman complained of urge incontinence for the past 1 month. Her medical history and family history were negative. She un- derwent a gastrectomy for stomach cancer (T3N0M0) 4 years previously and adjuvant chemotherapy with the FAM regimen, which combines 5-fluorouracil, doxorubicin, and mitomycin. The physical examination revealed no specific findings. Her serum creatinine on presentation was $0.6 \mathrm{mg} / \mathrm{dL}$. Her urine culture was negative. Anticholinergic medication was prescribed for 1 month. However, her urge incontinence was aggravated. Abdominal computerized tomography showed bladder wall thickening of the anterior bladder wall, suggesting hematogenous metastasis (Figure 1). She underwent cystoscopy, which revealed the presence of a suspicious bladder mucosal lesion (Figure 2). A biopsy of the bladder lesion was performed. The pathological analysis of the biopsy specimen revealed poorly differentiated metastatic signet-ring cell adenocarcinoma (Figure 3). She was transferred to the department of gastroenterology and under96

\footnotetext{
Corresponding Author: Yun Seob Song

Department of Urology, Soonchunhyang University Hospital, 657, Hannam-dong, Yongsan-gu, Seoul 140-743, Korea

Tel: 02-709-9375 / Fax: 02-790-2468 / E-mail: yssong@hosp.sch.ac.kr
}

Submitted: August 06, 2010 / Accepted (with revisions): August 19, 2010 


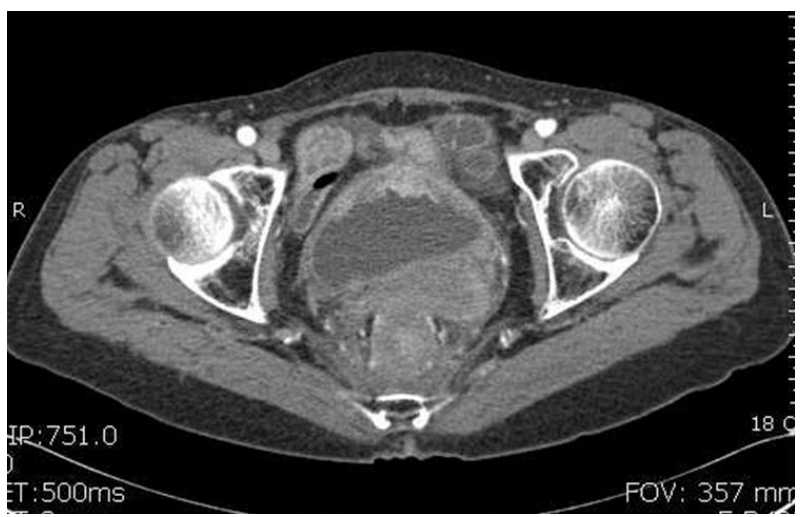

Figure 1. Pelvic computerized tomography showing thickened anterior bladder wall, suggesting tumoral infiltration.

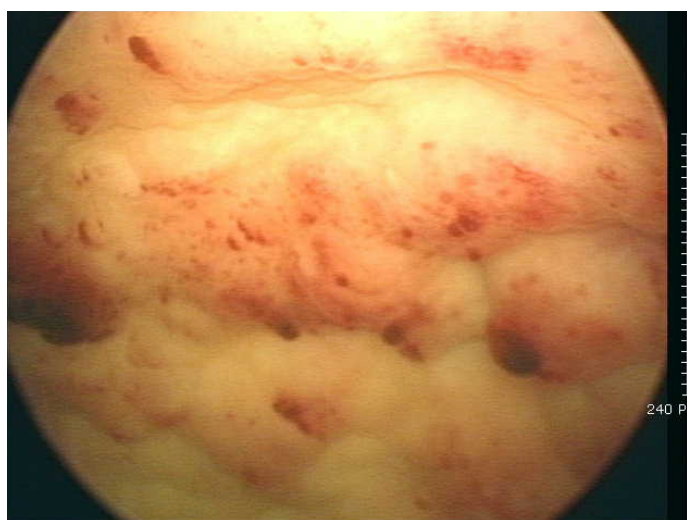

Figure 2. Cystoscopy that revealed a suspicious bladder mucosal lesion in the anterior bladder wall.

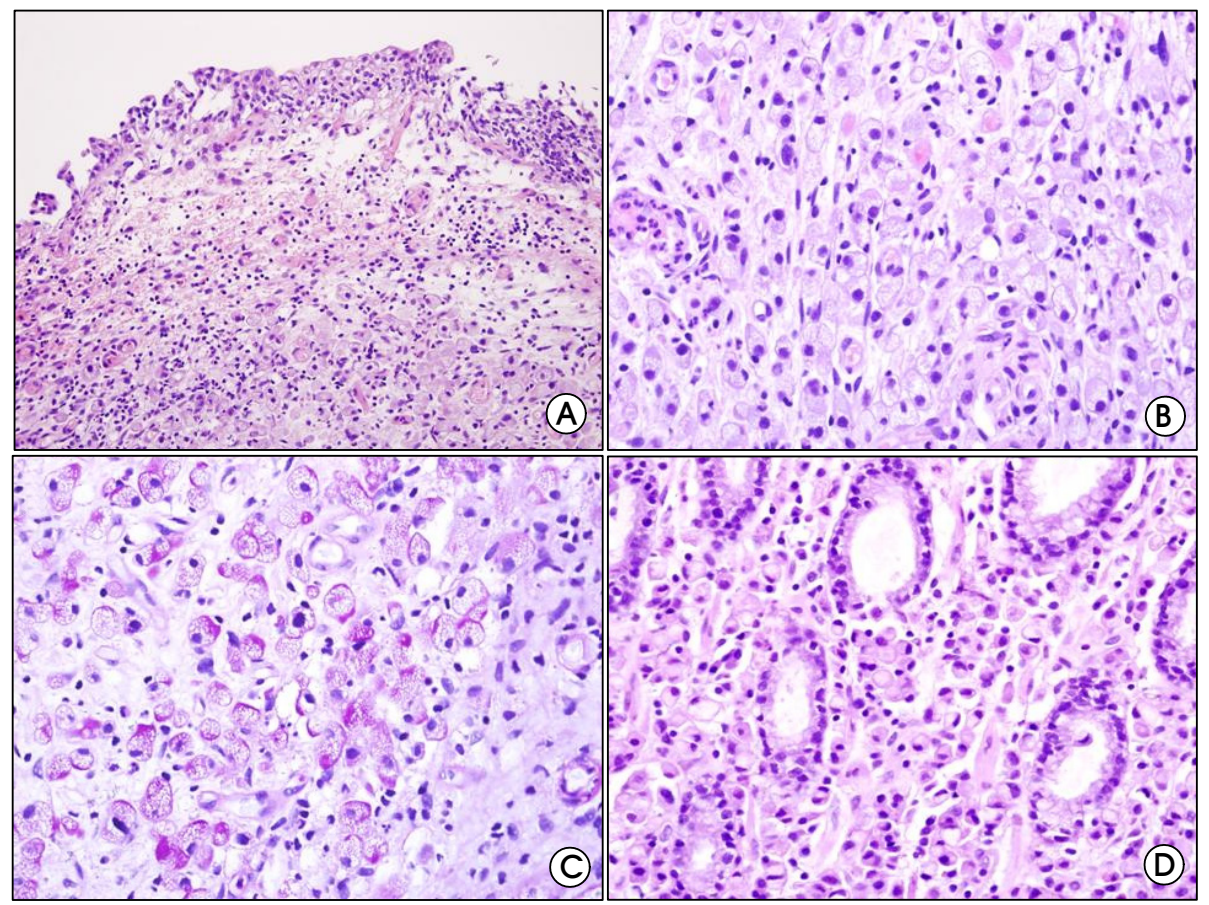

Figure 3. Photomicrography showing infiltration of signet-ring cell adenocarcinoma in anterior bladder wall. A: Beneath the urothelium with chronic inflammation, discohesive cellular infiltration is noted. (H\&E, $\times 200)$, B: Tumor cells disclose abundant vacuolated cytoplasm and eccentric nuclei. (H\&E, $\times 400)$, C: PAS and d-PAS stain reveal positive finding in the tumor cells.(d-PAS, $\times 400)$, D: In previously operated stomach, tumor cells between the gastric glands show features of tumor cells of urinary bladder, suggestive of metastasis from the stomach. $(H \& E, \times 400)$

went chemotherapy with a combined compound pharmaceutical agent containing tegafur, gimeracil, and oeteracil potassium (trade name: TS-1) and cisplatin.

\section{Discussion}

Stomach cancer metastases to the bladder are 
unusual but have been reported occasionally in the literature. The clinical presentation usually ranges from the more common macroscopic hematuria to irritative voiding symptoms such as urgency, nocturia, or dysuria. Although this symptom may be associated with bladder neoplasm, it is also very common in postmenopausal women. It may be helpful to look for even subtle urinary symptoms in these patients. In this case, the cystoscopic examination was prompted by a complaint of urinary urgency.

The bladder may be secondarily involved by cancers from another primary site. The most common primary sites are the prostate, ovary, uterus, colon and rectum, lung, breast, kidney, and stomach [3]. Bladder metastases of gastric carcinoma correspond to approximately $1 \%$ of all neoplasias affecting the bladder [1]. Among cases of metastatic bladder cancer published to date, the incidence is similar between genders, and the mean age ranges from 44 to 63 years [4]. Potential mechanisms that can contribute to the development of secondary bladder lesions are direct extension of the primary focus, implant of exfoliated cells from the ureter and renal pelvis, and lymphatic, hematogenic, or peritoneal dissemination from a distant focus [2].

In our patient, irritative urinary symptoms prompted the performance of complementary examinations such as cystoscopy and abdominal computerized tomography, which resulted in the diagnosis of a metastatic bladder tumor. Assessment of the bladder should be performed to rule out a concomitant condition that may cause or be associated with incontinence [5]. Bladder cancer may be a cause of urgency incontinence. In cases unresponsive to anticholinergic therapy, we must keep in mind the possibility of bladder cancer. Irritative urinary symptoms can be the first clinical manifestation in these patients.

\section{References}

1) Valero Puerta JA, Medina Perez M, Garcia Carriazo M, Valpuesta Fernandez I, Sanchez Gonzalez M, Mompean FO. Bladder metastasis of signet-ring cell adenocarcinoma from the stomach. Arch Esp Urol 2000;53:839-41

2) Leddy FF, Peterson NE, Ning TC. Urogenital linitis plastica metastatic from stomach. Urology 1992;39: 464-7

3) Messing ED. Urothelial tumors of the bladder. In Wein AJ, Kavoussi LR, Novick AC, Partin AW, Peters CA, editors. Capbell-Walsh Urology 8th ed. Philadelphia: Saunders; 2007;2407-46

4) Saba NF, Hoening DM, Cohen SI. Metastatic signet-ring cell adenocarcinoma to the urinary bladder. Acta Oncol 1997;36:219-20

5) Nitti VW, Blaivas JG. Urinary incontinence: Epidemiology, pathophysiology, evaluation, and management overview. In Wein AJ, Kavoussi LR, Novick AC, Partin AW, Peters CA, editors. Capbell-Walsh Urology 8th ed. Philadelphia: Saunders; 2007; 2046-90 